\title{
Perspective
}

\section{Efficacy of Repetitive Transcranial Magnetic Stimulation (rTMS) in the Treatment of Affective Disorders}

\author{
Thomas E Schlaepfer*, ${ }^{*, 2}$, Markus Kosel' and Charles B Nemeroff \\ 'Psychiatric Neuroimaging Group, Department of Psychiatry, University Hospital Bern, Switzerland; ${ }^{2}$ Department of Psychiatry, The Johns Hopkins \\ University School of Medicine, Baltimore, MD, USA; ${ }^{3}$ Department of Psychiatry and Behavioral Sciences, Emory University School of Medicine, \\ Atlanta, GA, USA
}

\begin{abstract}
Transcranial magnetic stimulation (TMS) is a relatively noninvasive technique to interfere with the function of small cortical areas through currents induced by alternating magnetic fields emanating from a handheld coil placed directly above the targeted area. This technique has clear effects on a whole range of measures of brain function and has become an important research tool in neuropsychiatry. More recently, TMS has been studied in psychiatry mainly to assess its putative therapeutic effects in treatment refractory major depression. Most studies indicate that both low-frequency TMS and higher $(20 \mathrm{~Hz})$ frequency repetitive TMS may have some antidepressant properties. However, definite therapeutic effects of clinical significance still remain to be demonstrated.

Neuropsychopharmacology (2003) 28, 20 I-205. doi:I 0.1038/sj.npp. I 300038
\end{abstract}

Keywords: major depression; transcranial magnetic stimulation

\section{PRINCIPLE OF TRANSCRANIAL MAGNETIC STIMULATION}

Transcranial magnetic stimulation (TMS) refers to a technique of delivery of magnetic pulses to the cortex using a handheld stimulating coil, which is directly applied to the head. The equipment necessary for delivering TMS consists basically of two parts: a stimulator that generates brief pulses of strong electrical currents whose frequency and intensity can be varied and a stimulation coil connected to the stimulator. The magnetic field generated at the coil passes unimpeded through scalp and skull and induces an electrical current in the underlying tissue, which depolarizes neurons. The main advantage of this stimulation method is its relative noninvasiveness and the possibility to stimulate very focused brain areas. With recent technology, single, paired or repetitive pulses can be delivered. Cortical excitability may be increased or decreased depending on the stimulation frequency (Hallett, 2000), and TMS has been shown to modify regional cerebral blood flow (Bohning et al, 2000; Catafau et al, 2001).

Barker first demonstrated the induction of muscle potentials by magnetic stimulation of the central nervous system in 1985 (Barker et al, 1985). He induced muscle twitching with a coil of $10 \mathrm{~cm}$ diameter placed on the scalp over the motor cortex. A brief pulse of $110 \mu$ s with a peak

\footnotetext{
*Correspondence: Dr TE Schlaepfer, Psychiatric Neuroimaging Group, Department of Psychiatry, University Hospital, 3010 Bern, Switzerland, E-mail: schlaepf@jhmi.edu

Received 30 May 2002; accepted 10 July 2002

Online publication: I August 2002 at htpp://www.acnp.org/citations/ Npp 080102361
}

current of $4000 \mathrm{~A}$ was applied and pulses at a maximal rate of $0.33 \mathrm{~Hz}$ were delivered.

With the possibility of stimulating the motor cortex noninvasively, TMS replaced high-voltage transcutaneous electrical stimulation previously used in clinical studies to measure variables such as central motor conduction time. Altered conduction time may be associated with a variety of neurological disorders such as multiple sclerosis, amyotrophic lateral sclerosis, cervical myelopathy and degenerative ataxic disorders. TMS has great potential in the intraoperative monitoring of the integrity of motor tracts during surgery of the brain and spinal tract (Murray, 1991). TMS has found widespread diagnostic use in neurology for demyelinating disorders involving the excitability and the connections of the motor cortex with other parts of the nervous system involved in motor function (Ziemann and Hallett, 2000).

In 1987, Bickford extended the field of TMS research into neuropsychiatry: he described transient mood elevation in several normal volunteers receiving single-pulse stimulations to the motor cortex (Bickford et al, 1987). This was the starting point of the scientific investigation of effects of depolarizing magnetic fields in a variety of neuropsychiatric disorders. Soon after, open studies of the effects of TMS on patients with major depression were conducted using single-pulse stimulations at frequencies less than $0.3 \mathrm{~Hz}$ (Grisaru et al, 1994; Höflich et al, 1993; Kolbinger et al, 1995). In these studies, relatively large areas under the vertex were stimulated bilaterally and involved only very few subjects. More recent work has suggested that slow and fast repetitive TMS (rTMS) may have some value in depression. 


\section{BASIC EFFECTS}

Magnetic fields generated by TMS are interacting with an extremely complex biological system where essential interactions between brain and mind take place (Kandel, 1998, 1999). It is obvious that the impact of these fields on brain systems is difficult to evaluate, because monitoring functions of the living human brain is only possible by assessing summation responses that are determined by the action of tens of thousands or more cells. The actual psychopathological models of psychiatric disorders are integrating the so-called functional systems at molecular, cellular, neurotransmitter, organ, systemic or individual and social levels that are not understood in detail. Presenting the mechanisms of action of TMS both as a research or treatment tool challenges old hypotheses of brain function and, hopefully, allows the creation of new ones. Several acute and chronic alterations at different levels, ranging from changes in gene expression of cells in the central nervous system to alterations in mood and behavior have been documented during and after the application of TMS.

Ji reported recently that one single train of rTMS applied to rats in vivo induced $\mathrm{c}$-fos and c-jun expression in different brain including key regions controlling circadian biological rhythms (Ji et al, 1998). Similar stimulation parameters have earlier been shown to posses efficacy in an animal model of depression (Fleischmann et al, 1995). These findings might point to a possible antidepressant mode of action of TMS effects via circadian rhythms. The finding that immediate-early gene expression is modified by TMS has been replicated and further examined both in vivo and in vitro (Doi et al, 2001; Hausmann et al, 2001).

Keck measured modulatory effects of frontal rTMS in rat brain in vivo using intracerebral microdialysis (Keck et al, 2000). There was a continuous reduction in arginine vasopressin release of up to $50 \%$ within the hypothalamic paraventricular nucleus. In contrast, the release of taurine, aspartate and serine was selectively stimulated within this nucleus. Furthermore, in the dorsal hippocampus the extracellular concentration of dopamine was elevated in response to rTMS.

By using PET scanning, a reduction in ${ }^{11} \mathrm{C}$ raclopride binding to dopamine receptors in the left dorsal caudate nucleus was observed in eight volunteers after left dorsolateral prefrontal cortex (DLPFC) rTMS. This implies that rTMS can trigger dopamine release in these brain structures (Strafellea et al, 2001).

Several studies documented the effect of rTMS on plasma levels of a variety of hormones including cortisol, prolactin and thyroid stimulating hormone. Results from these studies are inconclusive but indicate that TMS might significantly affect neuroendocrine function (Cohrs et al, 1998; George et al, 1996; Szuba et al, 2001).

TMS can transiently disrupt or induce activity in focal brain regions, depending on the region stimulated. Applied to the visual cortex for example, strong TMS can produce phosphenes and stimuli of lower intensity induce transient scotomas (Hallett, 2000). Moreover, other functions, such as linguistic processing, can be investigated with rTMS (Flitman et al, 1998). A neuromodulatory effect of subthreshold high-frequency rTMS has been observed in 10 subjects. After 1250 stimulations at $90 \%$ motor threshold, an intracortical inhibition could be measured, which persisted for at least $10 \mathrm{~min}$ after the rTMS stimulation (Peinemann et al, 2000).

The combination of noninvasive stimulation of the brain coupled with functional neuroimaging techniques offers novel opportunities to investigate human brain function. It also allows visualization of the effects of TMS including those distant from the site of stimulation (Paus et al, 1997). For example, 10 medication-free subjects suffering from major depression (eight unipolar, two bipolar), received in a crossover, randomized study rTMS at the left prefrontal cortex (LPFC), at $100 \%$ motor threshold, at either 20 or $1 \mathrm{~Hz}$. After $20 \mathrm{~Hz}$, an increase in regions cerebral blood flow $(\mathrm{rCBF})$ in the prefrontal cortex left $>$ right, cingulated gyrus left $\geqslant$ right, left amygdala, bilaterally insula, basal ganglia, hippocampus, parahippocampus, thalamus, cerebellum was observed, after $1 \mathrm{~Hz}$ only decreases in $\mathrm{rCBF}$ in right prefrontal cortex, left medial cortex, left basal ganglia and left amygdala was noted. Individuals whose depressive symptoms improved with one frequency worsened with the other (Speer et al, 2000).

The above-mentioned multidisciplinary results suggest that TMS has prominent and reproducible effects on the brain. This puts TMS apart from other putative approaches to treat neuropsychiatric disorders (Hallett, 2000). One problem shared with antidepressants is that the link between changes at cellular levels and complex behavioral changes - such as the ones observed in depression-has been very difficult to establish. The field of neuropsychiatric research in TMS has somewhat suffered from a 'top-down' approach in which early promising results in depression have led to an enthusiasm for clinical studies without sufficient basic neurobiologic rationale. Approaches integrating findings from all levels of brain-molecular to behavioral - systems are extremely important and should be undertaken in order to support ongoing clinical research.

\section{EFFECTS ON MOOD}

It is of obvious importance to elucidate the exact structural and functional bases of affect to understand the neurobiology of, and putative therapeutic interventions for, human disorders like depression and mania. Converging evidence from different areas of research support the hypothesis that mood is regulated by an interconnected network of brain regions encompassing prefrontal, cingulate, parietal, and temporal cortical regions as well as parts of the striatum, thalamus, and hypothalamus. Among them, the limbic system integrates external stimuli with internal drives and is part of a distributed neural network that marks stimuli and events with positive or negative value (Aggleton, 1993). Lesions of this network from tumor, infarction or transient disruption often result in mood changes. In addition, alterations of cerebral blood flow and metabolism in the dorsolateral, ventrolateral, orbitofrontal, and medial frontal regions, as well as the subgenual prefrontal and anterior cingulate cortex have been demonstrated in patients suffering from major depression (Mayberg, 1997; Soares and Mann, 1997). The prefrontal cortex has been implicated with mood generation and modulation, although this view is not universal (George et al, 1995a; Lane et al, 1997; Paradiso et al, 1997). 
rTMS has been used in healthy subjects to help elucidate the basic neurophysiology of mood generation and modulation by stimulating the DLPFC. Six studies are available in which the effect of prefrontal rTMS on mood systems in normal volunteers was investigated. In half of the studies, rTMS over the LPFC transiently induced a decrease in self-rated happiness and an increase of sadness compared to the effects of right prefrontal cortical rTMS (Dearing et al, 1997; George et al, 1996; Pascual-Leone et al, 1996). Three more recent studies (Cohrs et al, 1998; Mosimann et al, 2000; Nedjat et al, 1998) failed to demonstrate any effects on mood in healthy volunteers.

\section{EFFECTS ON MAJOR DEPRESSION}

Owing to its ability to interfere focally with neuronal circuits, rTMS has been proposed and subsequently researched as a putative therapeutic approach in refractory major depression (Nemeroff, 1996; Nestler, 1998). As in the studies of mood modulation by rTMS, the DLPFC has been the most important target for stimulation in major depression studies. George reported the first open study of the effects of rTMS in six patients with refractory depression treated with five daily rTMS sessions to the left DLPFC (George et al, 1995b). He reported that two patients in this study experienced improvement as assessed by a reduction of $26 \%$ in the Hamilton Rating Scale for Depression (HRSD) scores. Open and blinded studies of rTMS to the left DLPFC followed with varying results. A relatively large open study demonstrated that $42 \%$ of 56 patients responded to five daily rTMS sessions; the elderly exhibited a considerably lower response rate (Figiel et al, 1998). A 2 weeks treatment study resulted in a $41 \%$ decrease in HRSD scores in another open trial (Triggs et al, 1999). However, there are open studies that have failed to demonstrate any antidepressant activity of rTMS (Schouten et al, 1999).

In the sham-controlled, single-blinded studies of rTMS in treatment-resistant depression, effect sizes have varied considerably. In a within-subject crossover, sham-controlled study of 12 depressed patients treated for 2 weeks with stimulation to the left DLPFC only somewhat modest antidepressant efficacy of rTMS was found (George et al, 1997). In a more recent study, an antidepressant effect in 20 subjects that was statistically different from sham stimulation using similar stimulation parameters in a parallel design, but still only of modest clinical impact, was demonstrated (Berman et al, 2000). In some studies, a low stimulation intensity of $80 \%$ of motor threshold was used. Generally, it seems that higher intensity may be more effective, although Loo et al (1999) found no differences between active and sham rTMS using a much higher stimulation intensity ( $110 \%$ of motor threshold). In a large sham-controlled trial with 71 patients that utilized lowfrequency rTMS, it was demonstrated that $1 \mathrm{~Hz}$ stimulation to the right DLPFC was significantly more effective than sham stimulation (Klein et al, 1999). It is unclear whether stimulation of the left DLPFC at these parameters would have had the same effect. The effect of frequency was compared in a study in which 18 patients were randomized to single-pulse TMS, $10 \mathrm{~Hz}$ rTMS and sham rTMS delivered to the left DLPFC. A mild antidepressant effect with single- pulse TMS was demonstrated (Padberg et al, 1999). Recently, a sham-controlled trial in which 20 patients were randomly assigned to receive an equivalent number of pulses at $5 \mathrm{~Hz}$ or $20 \mathrm{~Hz}$ over 2 weeks was reported in which both active groups experienced a $45 \%$ reduction in depression severity ratings and none of the patients responded to the sham stimulation (George et al, 2000). This suggests that lower frequencies may have therapeutic efficacy as well, which is important because slow rTMS is associated with a lower seizure risk. An analysis of treatment response and cerebral metabolism suggests that patients with hypometabolism at baseline may respond better to high-frequency stimulation $(20 \mathrm{~Hz})$, whereas those with baseline hypermetabolism respond better to $1 \mathrm{~Hz}$ stimulation (Kimbrell et al, 1999). However, the effects of rTMS on mood examined in this study were not statistically significant.

There is some indication that TMS stimulation at higher amplitudes might be more efficacious. Recently, a negative correlation between the distance from the coil to the cortex and antidepressant response expressed as the percentage of HRSD rating decrease before and after treatment in a relatively older patient group with treatment refractory major depression was reported (Mosimann et al, 2002). This study demonstrated that there might be a process of prefrontal atrophy that outpaces motor cortex atrophy in chronically depressed middle-aged subjects. This observation together with the established fact that therapeutic seizures have a strong and reliable effect in depression lead to the development of another method that uses rTMS at convulsive levels as a more targeted form of convulsive therapy. Efficacy and side effects of electroconvulsive therapy (ECT) seem to be dependent upon the path of the current passed through the brain (Sackeim, 2000; Sackeim et al, 1993). Therefore targeting seizures to focal cortical areas, such as regions of the prefrontal cortex, may reduce some of the side effects of convulsive treatment. Magnetic seizure therapy (MST) has now been tested in proof of concept studies both in nonhuman primates and patients (Lisanby et al, 2001b) and preliminary results on cognitive side effects of the treatment compared to those of ECT have been obtained (Lisanby et al, 2001a). Much additional research is obviously needed to evaluate the putative clinical efficacy of this approach and to determine if it has significant advantages over ECT.

\section{DISCUSSION}

Today, data on clinical efficacy of rTMS in mood disorders are certainly not unequivocal but nevertheless interesting and encouraging. Rigorously controlled, double-blinded multicenter trials are needed in order to address adequately the question of the clinical efficacy of TMS. Prior to such studies, technical problems in the application of TMS have to be solved, for example, more satisfactory sham conditions must be developed. Today, using analogies to antidepressant drug development, valid phase II trials must be conducted. Crucial unanswered questions remain including the long-term efficacy of TMS, prevention of relapse and long-term side effects. The key findings in the acute treatment of depression have not been systematically 
replicated, and effect sizes have often been small and variable. Sources of variability across studies include differences in stimulation parameter settings, concomitant medications, and different characteristics of patient samples. In addition, simple and economical methods for precise and reproducible coil placement are needed, as this factor is likely to be important for effectiveness (Kozel et al, 2000). In much of this work, the magnitude of antidepressant effect, while often statistically significant, has been below the threshold of clinical usefulness (Berman et al, 2000) and has not lived up to expectations raised by encouraging results in animal studies. Furthermore, the persistence of antidepressant effects beyond the 1-2 week treatment period has rarely been examined. Initial evidence suggests that the beneficial effects may be transitory, making the development of maintenance strategies important if rTMS is to become clinically applicable.

Establishing whether nonconvulsive rTMS has antidepressant properties aside from its clinical usefulness is of theoretical importance, because positive data support the notion that focally targeted manipulations of cortical function can result in mood improvement. Nonetheless, the future of rTMS is far from certain in its usefulness as a clinical addition to the antidepressant armamentarium. A recent systematic review of published and unpublished studies on the effectiveness of rTMS in the treatment of refractory major depression demonstrated both a relative lack in the overall quality of studies (compared to drug registration trials) and the lack of a main effect (Martin et al, 2002).

There is no consensus about the exact mechanisms of action of how rTMS might induce antidepressant effects. However, this is also the case for other antidepressant treatments including ECT, bupropion and the SSRIs and SNRIs. Research on rTMS has mainly been empirical. There are many variables of rTMS application and a large parameter space has therefore to be carefully explored in order to find the most efficacious treatment. This process will most likely be slow, because there is little funding available for such studies. Nevertheless, rTMS has clearly effects on the brain - which is certainly remarkable - and it might be that rTMS is a treatment modality in search of a suitable application in psychiatry.

However, from the viewpoint of the neuroscientist, TMS is a methodology with great potential as a research tool (Hallett, 2000; Lisanby et al, 2000). This technique, by itself and combined with other methods such as EEG and neuroimaging, may be useful to test functional connectivity, neuroplasticity, information processing (eg in the visual system), indirect and direct motor control, and aspects of mood control. It affords testing of either general hypotheses of the function of the brain at different levels and hypotheses of the underlying pathology of neuropsychiatric disorders. Even if the early enthusiasm, which prevailed after early studies of clinical effects in the treatment of mood disorders settled down somewhat, rTMS will be even more useful as an investigational tool of basic and clinical research.

\section{ACKNOWLEDGEMENTS}

This work was supported by Grants 4038-044046 and 3231044523 from the Swiss National Science Foundation to Dr Schlaepfer.

\section{REFERENCES}

Aggleton JP (1993). The contribution of the amygdala to normal and abnormal emotional states. Trends Neurosci 16: 328-333.

Barker AT, Jalinous R, Freeston IL (1985). Noninvasive magnetic stimulation of human motor cortex. Lancet 2: 1106-1107.

Berman RM, Narasimhan M, Sanacora G, Miano AP, Hoffman RE, $\mathrm{Hu} \mathrm{XS}$ et al (2000). A randomized clinical trial of repetitive transcranial magnetic stimulation in the treatment of major depression. Biol Psychiatry 47: 332-337.

Bickford RG, Guidi M, Fortesque P, Swenson M (1987). Magnetic stimulation of human peripheral nerve and brain: response enhancement by combined magnetoelectrical technique. Neurosurgery 20: 110-116.

Bohning DE, Shastri A, Wasserman EM, Ziemann U, Lorberbaum JP, Nahas $\mathrm{Z}$ et al (2000). BOLD-fMRI response to single-pulse transcranial magnetic stimulation (TMS). J Magn Reson Imaging 11: 569-574.

Catafau A, Perez V, Gironell A, Martin J, Kulisevsky J, Estorch M et al (2001). SPECT mapping of cerebral activity changes induced by repetitive transcranial magnetic stimulation in depressed patients. A pilot study. Psychiatry Res 106: 151-160.

Cohrs S, Tergau F, Riech S, Kastner S, Paulus W, Ziemann U et al (1998). High-frequency repetitive transcranial magnetic stimulation delays rapid eye movement sleep. Neuroreport 9: 3439-3443.

Dearing J, George MS, Greenberg BD, Wassermann EM, Schlaepfer TE, Murphy DL et al (1997). Mood effects of prefrontal repetitive high frequency transcranial magnetic stimulation (rTMS) in healthy volunteers. CNS Spectrums 2: 53-68.

Doi W, Sato D, Fukuzako H, Takigawa M (2001). c-Fos expression in rat brain after repetitive transcranial magnetic stimulation. Neuroreport 12: 1307-1310.

Figiel GS, Epstein C, McDonald WM, Amazon-Leece J, Figiel L, Saldivia A et al (1998). The use of rapid-rate transcranial magnetic stimulation (rTMS) in refractory depressed patients. $J$ Neuropsychiatry Clin Neurosci 10: 20-25.

Fleischmann A, Prolov K, Abarbanel J, Belmaker RH (1995). The effect of transcranial magnetic stimulation of rat brain on behavioral models of depression. Brain Res 699: 130-132.

Flitman S, Grafman J, Wassermann E, Cooper V, O'Grady J, Pascual-Leone A, Hallett M (1998). Linguistic processing during repetitive transcranial magnetic stimulation. Neurology 50: 175181.

George MS, Ketter TA, Parekh PI, Horwitz B, Herscovitch P et al (1995a). Brain activity during transient sadness and happiness in healthy women. Am J Psychiatry 152: 341-351.

George M, Nahas Z, Molloy M, Speer A, Oliver N, Li X et al (2000). A controlled trial of daily left prefrontal cortex TMS for treating depression. Biol Psychiatry 48: 962-970.

George MS, Wassermann EM, Kimbrell TA, Little JT, Williams WE, Danielson AL et al (1997). Mood improvement following daily left prefrontal repetitive transcranial magnetic stimulation in patients with depression: a placebo-controlled crossover trial. Am J Psychiatry 154: 1752-1756.

George MS, Wasserman EM, Williams WA, Callahan A, Ketter TA, Basser P et al (1995b). Daily repetitive transcranial magnetic stimulation (rTMS) improves mood in depression. Neuroreport 6: $1853-1856$.

George MS, Wassermann EM, Williams WA, Steppel J, PascualLeone A, Basser P et al (1996). Changes in mood and hormone levels after rapid-rate transcranial magnetic stimulation (rTMS) of the prefrontal cortex. J Neuropsychiatry Clin Neurosci 8: 172180.

Grisaru N, Yaroslavsky Y, Abarbanel JM, Lamberg T, Belmaker R (1994). Transcranial magnetic stimultation in depression and schizophrenia. Eur Neuropsychopharmacol 4: 287-288.

Hallett M (2000). Transcranial magnetic stimulation and the brain. Nature 406: 147-150. 
Hausmann A, Marksteiner J, Hinterhuber H, Humpel C (2001). Magnetic stimulation induces neuronal c-fos via tetrodotoxinsensitive sodium channels in organotypic cortex brain slices in rat. Neurosci Lett 310: 105-108.

Höflich G, Kasper S, Hufnagel A, Ruhrmann S, Möller HJ (1993). Application of transcranial magnetic stimulation in the treatment of drug-resistant major depression: a report of two cases. Hum Psychopharmacol 8: 361-365.

Ji RR, Schlaepfer TE, Aizenman CD, Epstein CM, Qiu D, Huang JC et al (1998). Repetitive transcranial magnetic stimulation activates specific regions in rat brain. Proc Natl Acad Sci USA 95: $15635-15640$.

Kandel ER (1998). A new intellectual framework for psychiatry. Am J Psychiatry 155: 457-469.

Kandel ER (1999). Biology and the future of psychoanalysis: a new intellectual framework for psychiatry revisited. Am J Psychiatry 156: $505-524$.

Keck ME, Sillaber I, Ebner K, Welt T, Toschi N, Kaehler ST et al (2000). Acute transcranial magnetic stimulation of frontal brain regions selectively modulates the release of vasopressin, biogenic amines and amino acids in the rat brain. Eur J Neurosci 12: 37133720.

Kimbrell TA, Little JT, Dunn RT, Frye MA, Greenberg BD, Wassermann EM et al (1999). Frequency dependence of antidepressant response to left prefrontal repetitive transcranial magnetic stimulation (rTMS) as a function of baseline cerebral glucose metabolism. Biol Psychiatry 46: 1603-1613.

Klein E, Kreinin I, Chistyakov A, Koren D, Mecz L, Marmur S et al (1999). Therapeutic efficacy of right prefrontal slow repetitive transcranial magnetic stimulation in major depression: a doubleblind controlled study. Arch Gen Psychiatry 56: 315-320.

Kolbinger HM, Höflich G, Hufnagel A, Möller HJ, Kasper S (1995). Transcranial magnetic stimulation (TMS) in the treatment of major depression. Hum Psychopharmacol 10: 305-310.

Kozel FA, Nahas Z, de Brux C, Molloy M, Lorberbaum JP, Bohning D et al (2000). How coil-cortex distance relates to age, motor threshold, and antidepressant response to repetitive transcranial magnetic stimulation. J Neuropsychiatry Clin Neurosci 12: 376-384.

Lane RD, Reiman EM, Ahern GL, Schwartz GE, Davidson RJ (1997). Neuroanatomical correlates of happiness, sadness, and disgust. Am J Psychiatry 154: 926-933.

Lisanby S, Luber B, Barroilhet L, Neufeld E, Schlaepfer T, Sackeim $\mathrm{H}$ (2001a). Magnetic seizure therapy (MST): acute cognitive effects of MST compared with ECT. JECT 17-77.

Lisanby SH, Luber B, Peren T, Sackeim HA (2000). Transcranial magnetic stimulation: application in basic neuroscience and neuropsychopharmacology. Int J Neuropsychopharmacol 3: 259273.

Lisanby SH, Schlaepfer TE, Fisch HU, Sackeim HA (2001b). Magnetic seizure therapy of major depression. Arch Gen Psychiatry 58: 303-305.

Loo C, Mitchell P, Sachdev P, McDarmont B, Parker G, Gandevia S (1999). Double-blind controlled investigation of transcranial magnetic stimulation for the treatment of resistant major depression. Am J Psychiatry 156: 946-948.

Martin J, Barbanoj M, Schlaepfer T, Clos S, Perez V, Kulisevsky J et al (2002). Transcranial magnetic stimulation for treating depression, (Cochrane Review). Update Software, The Cochrane Library: Oxford.

Mayberg HS (1997). Limbic-cortical dysregulation: a proposed model of depression. J Neuropsychiatry Clin Neurosci 9: 471-481.

Mosimann U, Marré SC, Werlen S, Schmitt W, Hess CW, Fisch HU et al (2002). Antidepressant effects of repetitive transcranial magnetic stimulation in the elderly - Correlation between effect size and coil-cortex distance. Arch Gen Psychiatry 59: 560-563.

Mosimann UP, Rihs TA, Engeler J, Fisch HU, Schlapfer TE et al (2000). Mood effects of repetitive transcranial stimulation
(rTMS) of left prefrontal cortex in healthy volunteers. Psychiatry Res 94: 251-256.

Murray NMF (1991). Magnetic stimulation of cortex: clinical applications. J Clin Neurophysiol 8: 66-76.

Nedjat S, Folkerts HW, Michael ND, Arolt V (1998). Evaluation of the side effects after rapid-rate transcranial magnetic stimulation over the left prefronatl cortex in normal volunteers. Electroencephalogr Clin Neurophysiol 107: 96.

Nemeroff C (1996). Augmentation strategies in patients with refractory depression. Depress Anxiety 4: 169-181.

Nestler E (1998). Antidepressant treatments in the 21st century. Biol Psychiatr 44: 526-533.

Padberg F, Zwanzger P, Thoma H, Kathmann N, Haag C, Greenberg BD et al (1999). Repetitive transcranial magnetic stimulation (rTMS) in pharmacotherapy-refractory major depression: comparative study of fast, slow and sham rTMS. Psychiatry Res 88: 163-171.

Paradiso S, Robinson RG, Andreasen NC, Downhill JE, Davidson RJ, Kirchner PT et al (1997). Emotional activation of limbic circuitry in elderly normal subjects in a PET study. $A m \mathrm{~J}$ Psychiatry 154: 384-389.

Pascual-Leone A, Catala MD, Pascual-Leone Pascual A (1996). Lateralized effect of rapid-rate transcranial magnetic stimulation of the prefrontal cortex on mood. Neurology 46: 499-502.

Paus T, Jech R, Thompson CJ, Comeau R, Peters T, Evans AC (1997). Transcranial magnetic stimulation during positron emission tomography: a new method for studying connectivity of the human cerebral cortex. J Neurosci 17: 3178-3184.

Peinemann A, Lehner C, Mentschel C, Münchau A, Conrad B, Siebner HR (2000). Subthreshold $5-\mathrm{Hz}$ repetitive transcranial magnetic stimulation of the human primary motor cortex reduces intracortical paired-pulse inhibition. Neuroscience Letters 296: 21-24.

Sackeim HA (2000). Repetitive transcranial magnetic stimulation: what are the next steps? Biol Psychiatry 48: 959-961.

Sackeim HA, Prudic J, Devanand DP, Kiersky JE, Fitzsimons L, Moody BJ et al (1993). Effects of stimulus intensity and electrode placement on the efficacy and cognitive effects of electroconvulsive therapy. $N$ Engl J Med 328: 839-846.

Schouten EA, D'Alfonso AA, Nolen WA, De Haan EH, Wijkstra J, Kahn RS (1999). Mood improvement from transcranial magnetic stimulation. Am J Psychiatry 156: 669-670.

Soares JC, Mann JJ (1997). The anatomy of mood disordersreview of structural neuroimaging studies. Biol Psychiatry 41: 86-106.

Speer AM, Kimbrell TA, Wasserman EM, Repella JD, Willis MW, Herscovitch $\mathrm{P}$ et al (2000). Opposite effects of high and low frequency rTMS on regional brain activity in depressed patients. Biol Psychiatry 48: 1133-1141.

Strafellea AP, Paus T, Barrett J, Dagher A (2001). Repetitive transcranial magnetic stimulation of the human prefrontalt cortex induces dopamine release in the caudate nucleus. $J$ Neurosci 21: RC1571-RC1574.

Szuba MP, O’Reordon JP, Rai AS, Snyder-Kastenberg J, Amsterdam JD, Gettes DR et al (2001). Acute mood and thyroid stimulating hormone effects of transcranial magnetic stimulation in major depression. Biol Psychiatry 50: 22-27.

Triggs WJ, McCoy KJM, Greer R, Rossi F, Bowers D, Kortenkamp S et al (1999). Effects of left frontal transcranial magnetic simulation on depressed mood, cognition, and corticomotor threshold. Biol Psychiatry 45: 1440-1445.

Ziemann U, Hallett M (2000). Basic neurophysiological studies with TMS. In: George MS, Belmaker RH (eds). Transcranial Magnetic Stimulation in Neuropsychiatry. American Psychiatric Press: Washington DC. 